13. P.P. Yupapin, P. Saeung, and C. Li, Characteristics of complementary ring-resonator add/drop filters modeling by using graphical approach, Opt Commun 272 (2007), 81-86.

14. P.P. Yupapin and W. Suwancharoen, Chaotic signal generation and cancellation using a microring resonator incorporating an optical add/drop multiplexer, Opt Commun 280 (2007), 343-350.

15. L. Yuan, Z. Liu, J. Yang, and C. Guan, Twin-core fiber optical tweezers, Opt Exp 16 (2008), 4559-4566.

16. N. Malagninoa, G. Pescea, A. Sassoa, and E. Arimondo, Measurements of trapping efficiency and stiffness in optical tweezers, Opt Commun 214 (2002), 15-24.

17. S. Suchat, W. Khannam, and P.P. Yupapin, Quantum key distribution via an optical wireless communication link for telephone network, Opt Eng Lett 46 (2007), 100502-1-5.

18. P.P. Yupapin and S. Suchat, Entangle photon generation using fiber optic Mach-Zehnder interferometer incorporating nonlinear effect in a fiber ring resonator, J Nanophotonics 1 (2007), 13504-1-7.

(C) 2010 Wiley Periodicals, Inc.

\section{ANALYSIS OF TUNABLE FLAT DISPERSION OF GERMANIUM DOPED FIBER WITH LIQUID CLADDING}

\author{
Y. Liu, ${ }^{1}$ N. Chen, ${ }^{2}$ Y. Lai, ${ }^{2}$ and S. $\mathrm{Chi}^{2}$ \\ ${ }^{1}$ Department of Electrical and Computer Engineering, Texas A\&M \\ University, TX; Corresponding author: vaki@tamu.edu \\ ${ }^{2}$ Department of Photonics, National Chiao Tung University, \\ Hsinchu, Taiwan
}

Received 1 September 2009

ABSTRACT: We demonstrate numerically the tunable dispersionflattened fibers can be achieved by immersing the Ge-doped fiber core in different liquid claddings. The dispersion slope can be flattened over 1100-1700 nm, which is suitable for tunable supercontinuum generation with pumping wavelength in fiber communication or other wavelength regions. Finally, we demonstrate the feasibility to fabricate the proposed structure with chemical etching method. (C) 2010 Wiley Periodicals, Inc. Microwave Opt Technol Lett 52: 1357-1359, 2010; Published online in Wiley InterScience (www.interscience.wiley.com). DOI 10.1002/ mop.25209

Key words: optical waveguide; fibers; dispersion

\section{INTRODUCTION}

Supercontinuum (SC) light source is useful for biomedical investigation (e.g., optical tomography coherence), spectrum analysis, frequency metrology, and so forth. The SC generation is related to the complex processes including self phase modulation, cross phase modulation, stimulated Raman scattering, and four-wavemixing. Accordingly, the high nonlinearity of material and the low group velocity dispersion (GVD) near the pump wavelength are crucial to the generation efficiency and bandwidth of the SC. In optical fibers, a high nonlinear coefficient can be achieved by increasing the nonlinearity of core material such as with high Gedoping [1] or by improving the index contrast to obtain a better power confinement [2]. On the other hand, a low GVD near the pump wavelength had been succeeded by moving the zero-dispersion wavelength (ZDW) toward 800 and $1550-\mathrm{nm}$ wavelength to close to the high-peak-power pump wavelength for tapered fiber [2] and the photonic crystal fiber [3], respectively. However, the high dispersion slope near the ZDW of silica fibers gives rise to a very limiting useful bandwidth as the silica fiber has a very high phonon energy with its ZDW occurring at around $1300 \mathrm{~nm}$. More recently, the dispersive liquid is proposed to surround the tapered fiber to engineer fiber's dispersion and a broadband flattened GVD can be achieved over 1200-1400 nm [4, 5]. However, the high-peak-power femto-second mode-locked fiber laser at 1550$\mathrm{nm}$ wavelength band (e.g., $\mathrm{Er}^{3+}$-doped fiber laser) can not be used to produce SC with a high generation efficiency and a wide bandwidth.

In this article, we investigated numerically and concluded that Cargille index liquids surrounding the core of the high $\mathrm{Ge}-$ doped $(40 \mathrm{~mol} \%)$ single-mode fiber (SMF) can obtain a broadband flattened GVD over 1100-1700 nm with core diameter of $3 \mu \mathrm{m}$. The high Ge-doped SMF with the Cargille liquid cladding $\left(n_{\mathrm{D}}=1.42\right)$, which is much less dispersive than silica, can cause the ZDW moving toward $1550 \mathrm{~nm}$ and have a broadband flattened GVD over $600 \mathrm{~nm}$; this indicates high efficient SC can be generated in optical communication region. It was also found that the ZDW is tunable for from 900 to $1200-\mathrm{nm}$ wavelength with a flat dispersion by changing the index of liquid cladding with fixed core diameter, which means the tunable SC generation can be achieved more easily by adjusting the ZDW to the pumping wavelength with fixed fiber fabrication process and different liquid cladding.

\section{ANALYSIS FOR THE GVD OF HIGH Ge-DOPED FIBER CORE WITH LIQUID CLADDING}

Figure 1 shows the schematic diagram of the Ge-doped fiber core with liquid cladding. The amount of Ge-doping, waveguide diameter $d$, and index of liquid cladding are key parameters for broadband flattened GVD and wide bandwidth SC. The GVD is defined as the second derivative of propagation constant and is obtained by solving the dispersion equation for $\mathrm{HE}_{11}$ mode of optical fiber [4]. At the beginning, the diameter of the Ge-doped fiber core was set to be $2.5 \mu \mathrm{m}$ with the Cargille liquid cladding of $n_{\mathrm{D}}=1.40$. The dispersion curves shown in Figure 2(a) shows that high Ge-doping can make the ZDW move to longer wavelengths. Figure 2(b) shows that the $40 \mathrm{~mol} \% \mathrm{Ge}$-doped fiber with $3 \mu \mathrm{m}$ in core diameter has more flattened dispersion than others over 1100-1700 nm. Finally, different Cargille liquids were used as the cladding of the $40 \mathrm{~mol} \% \mathrm{Ge}$-doped fiber with the core diameter of $3 \mu \mathrm{m}$ to refine the GVD. The high Ge-doping provides higher nonlinearity than standard fibers. It is found that the Cargille liquid with $n_{\mathrm{D}}=1.42$ can achieve a broadband flattened GVD over 1100-1700 nm with the corresponding dispersion of ranging from +5 to $-9 \mathrm{ps} / \mathrm{nm} / \mathrm{km}$, as shown in Figure 3(a). The higher index contrast using lower index Cargill liquid though can obtain stronger power confinement but shows a higher dispersion at the wavelengths longer than $1000 \mathrm{~nm}$. This is because the higher index contrast can strongly confines the pump wavelength in silica fiber core and thus the dispersion is dominated by the high dispersive silica. When the evanescent wave is substantially

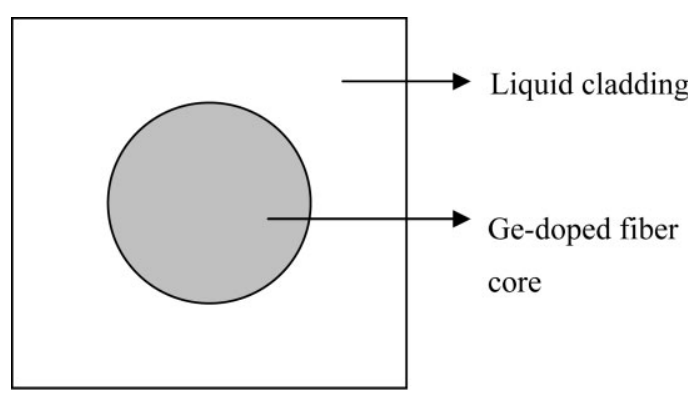

Figure 1 Schematic diagram of the proposed Ge-doped fiber core with liquid cladding 

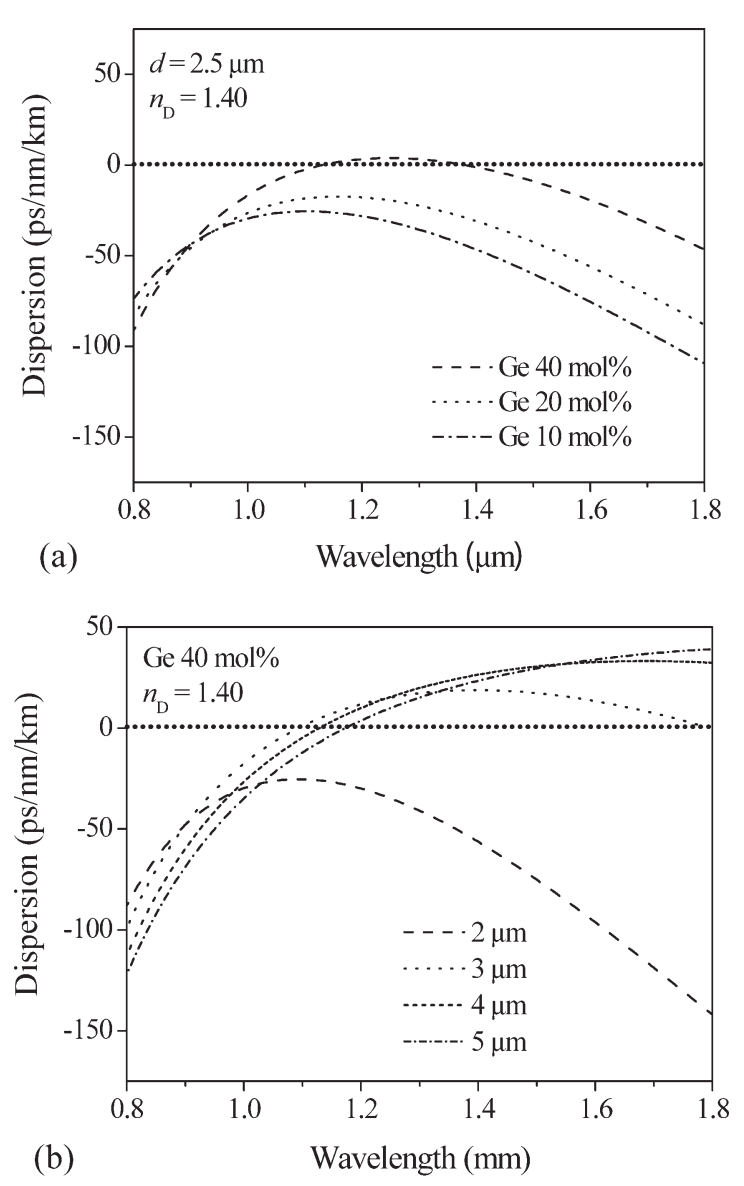

Figure 2 Calculated dispersion of (a) etched fibers with Cargille liquid $\left(n_{\mathrm{D}}=1.40\right)$ cladding for different Ge-doping and (b) etched fibers with $40 \mathrm{~mol} \% \mathrm{Ge}$-doping for different diameters

extended to the Cargille liquid cladding, the dispersion is significantly flattened as the liquid is much less dispersive than silica. Figure 3(b) is the calculated GVD of the tapered SMF fiber in air versus wavelength for the SC generation published in [2]; for tapered waist diameter $2.5 \mu \mathrm{m}$ the flattest GVD is below $200 \mathrm{ps} /$ $\mathrm{nm} / \mathrm{km}$ from 400 to $1500 \mathrm{~nm}$. Compare the GVD in Figure 3(a) with that in Figure 3(b), the $40 \mathrm{~mol} \%$ Ge-doped fiber cores immense in different liquid indices have GVD around $50 \mathrm{ps} / \mathrm{nm} /$ $\mathrm{km}$ or less from 800 to $1800 \mathrm{~nm}$, which is much flatter then that of the Ge-doped core or tapered fibers published in [2] in air. For the above reason, it is expected that $40 \mathrm{~mol} \%$ Ge-doped fiber core with liquid cladding can provide better efficiency for SC generation than tapered fiber in air. According to Figure 3(b), within the range of $1100-1700 \mathrm{~nm}$, many lasers can be used to generate SC with a high efficiency especially when the pumping wavelength is selected at the center of about $1400 \mathrm{~nm}$, the SC is expected to have the widest bandwidth. Besides, by changing the index of liquid cladding, the ZDW is tunable from 900 to 1200 $\mathrm{nm}$ wavelength and keep a flat dispersion; this can provide the tunable SC generation by matching the ZDW with the pumping wavelength in the range from 900 to $1200-\mathrm{nm}$ wavelength with a fixed fiber core diameter, which is easier to fix the fabrication process than fiber taping.

\section{FEASIBILITY FOR THE FABRICATION OF THE HIGH Ge-DOPED FIBER CORE WITH LIQUID CLADDING}

Here, we demonstrate the feasibility of the fabrication of our proposed structure. In fabrication, the $40 \mathrm{~mol} \%$ high Ge-doping fiber (HNA15A8: Prime Optical Fiber Corporation) used in this work
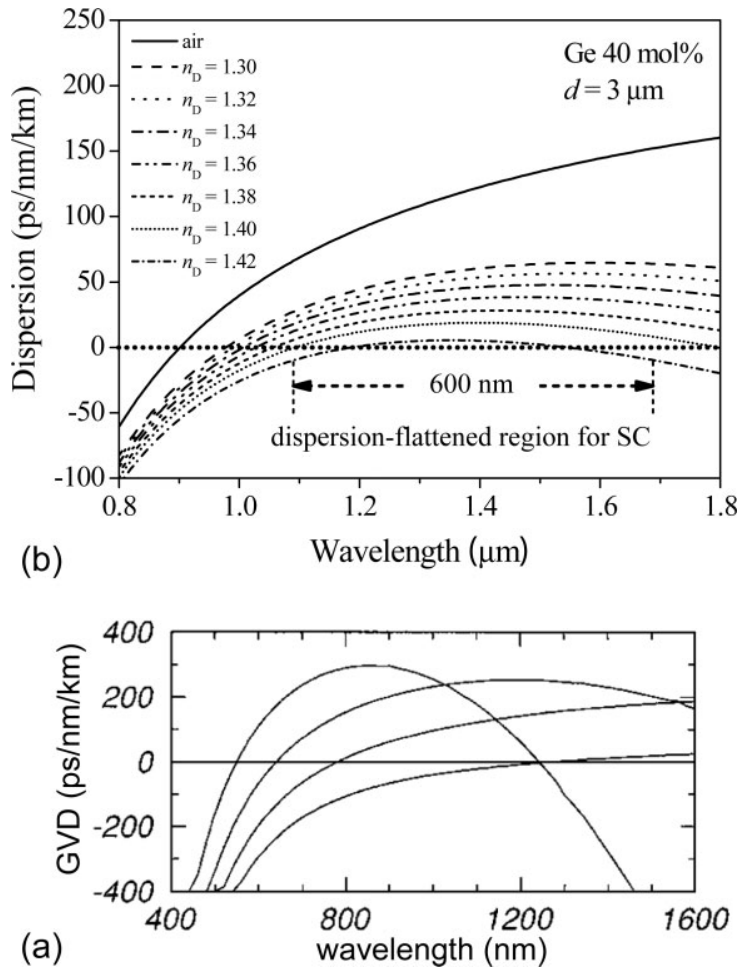

Figure 3 (a) Calculated dispersion of etched fiber with different Cargille liquids. (b) Calculated GVD of a taper waist in air versus wavelength for (left to right) diameters of $1.0,1.5$, and $2.5 \mu \mathrm{m}$ published in [2]. (c) Output spectra for waist diameters and average powers of 2.5 $\mu \mathrm{m}$ and $430 \mathrm{~mW}$ published in [2]. Reprinted with permission from Birks et al., Opt Lett 25 (2000), 1415-1417

has a core and cladding diameter of 2.9 and $80 \mu \mathrm{m}$ respectively. To access the evanescent wave region of the fiber, the cladding must be removed until the remained cladding thickness is below $1 \mu \mathrm{m}$. As the high Ge-doping must be kept in core and the processed fiber must be uniform enough to have the same diameter through the entire length of the fiber, chemical etching obviously surpasses the fused-tapering method to satisfy the above requirements. The fiber was unjacketed for 9-cm-long and fixed in a 12 -cm-long channel on an acrylate plate. Subsequently, it was immersed in the $45 \%$ hydrofluoric acid for $4 \mathrm{~h}$ and $50 \mathrm{~min}$ at $25^{\circ} \mathrm{C}$. The final diameter was measured to be around $3.2 \mu \mathrm{m}$

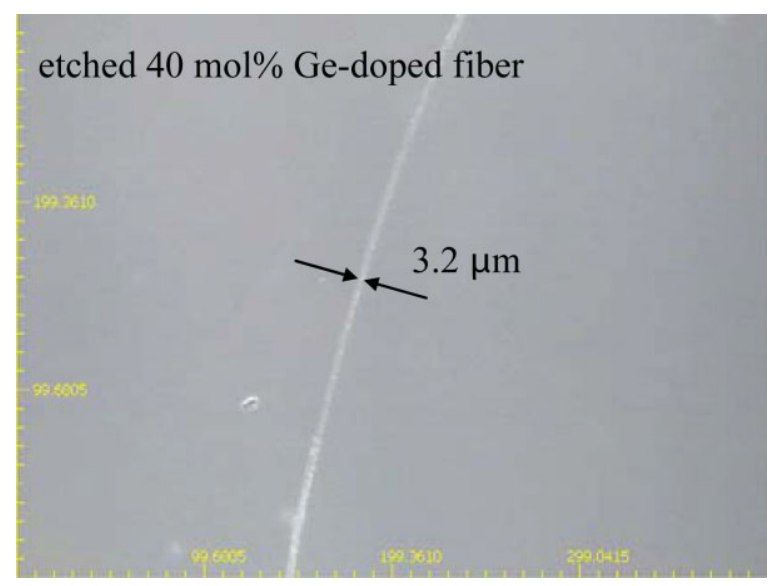

Figure $4 \mathrm{CCD}$ microscope image of the etched high Ge-doped (40 mol\%) fiber. [Color figure can be viewed in the online issue, which is available at www.interscience.wiley.com] 
under a $1000 \times$ CCD microscope shown in Figure 4. A $633 \mathrm{~nm}$ $\mathrm{He}-\mathrm{Ne}$ laser light was launched into this etched fiber and the light scattering from the etched region is negligible, which means that the etched surface does not induce serious scattering loss.

\section{CONCLUSIONS}

We have demonstrated numerically a broadband flattened and tunable GVD over $900-1800 \mathrm{~nm}$ by using the $40 \mathrm{~mol} \% \mathrm{Ge}-$ doped fiber core with different Cargille liquid cladding. The high Ge-doping can move the ZDW toward longer wavelengths, and the liquid cladding can flatten the total dispersion over a wide range. With this property, we expect that the wideband supercontinuum generation is achievable at fiber communication region and the tunable supercontinuum generation also can be accomplished by changing the liquid cladding to match the ZDW with the pumping wavelength for a fixed core diameter. At the end, the etching method was demonstrated to show the feasibility to fabricated the proposed structure.

\section{REFERENCES}

1. F.A. Oguama, A.M. Johnson, and W.A. Reed, Measurement of the nonlinear coefficient of telecommunication fibers as a function of $\mathrm{Er}, \mathrm{Al}$, and $\mathrm{Ge}$ doping profiles by using the photorefractive beamcoupling technique, J Opt Soc Am B 22 (2005), 1600-1604.

2. T.A. Birks, W.J. Wadsworth, and P. St. J. Russell, Supercontinuum generation in tapered fibers, Opt Lett 25 (2000), 1415-1417.

3. W.H. Reeves, J.C. Knight, P. St. J. Russell, and P.J. Roberts, Demonstration of ultra-flattened dispersion in photonics crystal fibers, Opt Express 10 (2002), 609-613.

4. R. Zhang, J. Teipel, X. Zhang, D. Nau, and H. Giessen, Group velocity dispersion of tapered fibers immersed in different liquids, Opt Express 12 (2004), 1700-1707.

5. C.M.B. Cordeiro, W.J. Wadsworth, T.A. Birks, and P. St. J. Russell, Engineering the dispersion of tapered fibers for supercontinuum generation with a $1064 \mathrm{~nm}$ pump laser, Opt Lett 30 (2005), 1980-1982.

(C) 2010 Wiley Periodicals, Inc.

\section{DUAL-BAND DESIGN OF SINGLE-STUB IMPEDANCE MATCHING NETWORKS WITH APPLICATION TO DUAL-BAND STUBBED T-JUNCTIONS}

Myun-Joo Park and Byungje Lee

Department of Wireless Communications Engineering, Kwangwoon University, 447-1 Wolgye-dong, Nowon-ku, Seoul 139-701, Korea;

Corresponding author: mjpark@kw.ac.kr

\section{Received 2 September 2009}

ABSTRACT: A new design method of single-stub impedance matching networks is presented for the dual-band application. The dual-band operating conditions are analyzed in detail and the design equations are derived in concise form for different locations and termination types of the stub line. The proposed impedance matching scheme is successfully applied to the design of a stubbed dual-band T-junction power divider. (C) 2010 Wiley Periodicals, Inc. Microwave Opt Technol Lett 52: 1359-1362, 2010; Published online in Wiley InterScience (www.interscience.wiley.com). DOI 10.1002/mop.25201

Key words: impedance matching; dual-band; T-junction

\section{INTRODUCTION}

Impedance matching is one of the most important and basic circuit operations in various microwave/RF applications. There are several methods available for the impedance matching purpose in general. In distributed circuit applications, the impedance matching strategies with the quarterwave lines or the single/double stub topologies are well established [1]. However, these basic topologies are limited to single frequency operation, and, recently, several studies are reported regarding dual- or multiband operation of impedance matching networks.

So far, most of the dual-band impedance transformers are based on the two-section cascaded transmission line structure [2-5]. They are basically a special case of the Chebyshev multisection transformers with equal-length transmission line sections [2-4]. Later, this method is extended to the unequal two-section topology for the dual-band matching of the complex impedances [5].

The single-stub impedance matching network is one of the most simple and basic topologies with the well-established design methodology [1]. However, it has not been studied yet for the dual-band applications. Only, the stub based harmonic matching network is proposed in [6] to synthesize desired impedances at a fundamental frequency and its second and third harmonics for the power amplifier applications.

This article proposes a new design method of the single-stub impedance matching network for the purpose of the dual-band operation. A careful analysis of the traditional single-stub topology reveals that it can be easily adapted to the dual-band operation with the careful selection of the lengths and impedances of the transmission line section and the stub line. It requires no additional circuits for the dual-band operation and the structural simplicity of the single-stub topology is fully preserved using single transmission line section and a stub line.

\section{STRUCTURE AND ANALYSIS}

\subsection{Dual-Band Design of Single-Stub Impedance}

Matching Networks

Figure 1 shows the two possible topologies for the single-stub impedance matching networks. Both of them consist of a transmission line section with a shunt stub line either at the source end [Fig. 1(a)] or at the load end [Fig. 1(b)]. All the impedance

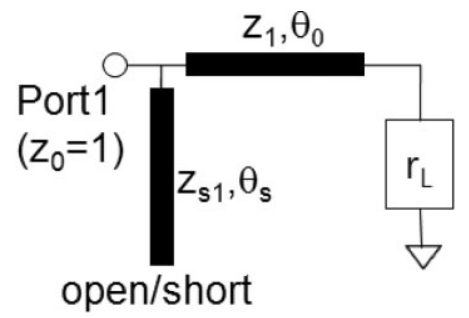

(a)

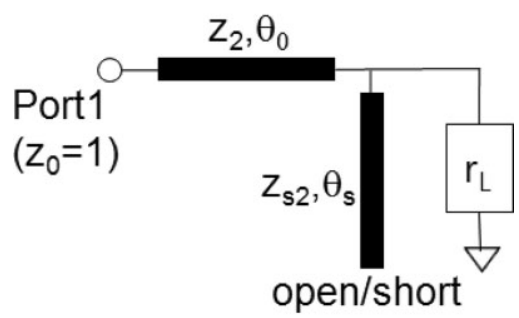

(b)

Figure 1 Single-stub impedance matching networks for dual-band applications. (a) Stub at the source end and (b) stub at the load end 